\title{
Correlation between structural properties and optical amplification in InGaN/GaN heterostructures grown by molecular beam epitaxy
}

\author{
A. Kaschner ${ }^{1}$, J. Holst ${ }^{1}$, U. von Gfug ${ }^{1}$, A. Hoffmann ${ }^{1}$, F. Bertram ${ }^{2}$, T. Riemann ${ }^{2}$, \\ D. Rudloff ${ }^{2}$, P. Fischer ${ }^{2}$, J. Christen ${ }^{2}$, R. Averbeck ${ }^{3}$, and H. Riechert ${ }^{3}$ \\ ${ }^{1}$ Institut für Festkörperphysik, TU Berlin, Hardenbergstrasse 36, 10623 Berlin, Germany \\ ${ }^{2}$ Institut für Experimentelle Physik, Otto-von-Guericke-Universität, PO Box 4120, \\ 39016 Magdeburg, Germany \\ ${ }^{3}$ Infineon Technologies, Corporate Research, CPR 7, 81730 München, Germany
}

\begin{abstract}
We comprehensively studied $\mathrm{InGaN} / \mathrm{GaN}$ heterostructures grown by molecular beam epitaxy (MBE) using a variety of methods of optical spectroscopy, such as cathodoluminescence microscopy (CL), time-integrated and time-resolved photoluminescence. To correlate the fluctuations in emission wavelength with values for the optical amplification we performed gain measurements in edge-stripe geometry. The lateral homogeneity can be drastically improved using a template of GaN grown on the sapphire substrate by metal-organic vapor phase epitaxy (MOVPE). Gain values up to $62 \mathrm{~cm}^{-1}$ were found in samples with low indium fluctuations, which is comparable to values for high-quality InGaN/GaN heterostructures grown by MOVPE.
\end{abstract}

\section{Introduction}

Heterostructures of compound group-III nitride-semiconductors are of great importance for the rapidly increasing market of optoelectronical devices in the blue spectral range [1]. While most articles in this field report on investigation of samples grown by metal-organic vapor phase techniques [2-4] we focus here on $\mathrm{InGaN}$ grown by MBE. It is known that fluctuations in the Indium concentration as well as the miscibility gap [5], are major issues concerning the optical properties of the InGaN alloy system. Recently, the formation of InN quantum dots [6] or InGaN with potential fluctuations [7] was suggested to be the origin of the luminescence in the material. The degree of Indium fluctuations in MBE-grown heterostructures can drastically be reduced by using a template of MOVPE-GaN on the sapphire substrate, which leads to improved optical properties and gain characteristics. Due to the high Indium desorption rate it is necessary to grow at temperatures typically below $700^{\circ} \mathrm{C}$ during $\mathrm{MBE}$ growth, which is far away from the thermodynamic equilibrium. However, we show that high-quality InGaN/GaN heterostructures can be grown under these conditions exhibiting gain values up to $62 \mathrm{~cm}^{-1}$ at $7 \mathrm{~K}$.

\section{Experimental Details}

Time-integrated photoluminescence experiments at different temperatures were performed using the $325 \mathrm{~nm}$ line of a He-Cd Laser. For time-resolved measurements a single photon counting setup was used with a 50 ps FWHM response to the laser pulse. A frequency-doubled dye laser pumped by the third harmonic of a $\mathrm{Nd}$ :YAG was used for excitation. The photoluminescence signal was analyzed in a $0.35 \mathrm{~m}$ subtractive double spectrometer and detected by a microchannel plate photomultiplier. The setup for the CL 
microscopy experiments is described in Ref. [8]. For the time integrated high-excitation investigations we used a dye laser pumped by an excimer laser, providing pulses with a duration of $15 \mathrm{~ns}$ at a rate of $30 \mathrm{~Hz}$ and a total energy of up to $20 \mu \mathrm{J}$ at $340 \mathrm{~nm}$. The samples were mounted in a bath cryostat at $1.8 \mathrm{~K}$. Gain measurements were performed using the variable-stripe-length method [9].

In this paper we focus on four typical $\mathrm{InGaN} / \mathrm{GaN}$ heterostructures which are chosen from a variety of samples. All samples are grown on sapphire, followed by a $1.8 \mu \mathrm{m} \mathrm{GaN}$ (MOVPE) layer for samples A-C and capped by a $30 \mathrm{~nm} \mathrm{GaN} \mathrm{layer.} \mathrm{Samples} \mathrm{A} \mathrm{and} \mathrm{B} \mathrm{are}$ double heterostructures including a $40 \mathrm{~nm}$ thick InGaN layer, with an Indium concentration of $10 \%$ and $13.7 \%$, respectively, as determined by XRD [10]. Sample C is a $10 x 5 \mathrm{~nm}$ InGaN multiple-quantum well (MQW) with $4 \mathrm{~nm} \mathrm{GaN} \mathrm{barriers.} \mathrm{The} \mathrm{growth}$ conditions for the InGaN wells were the same as for sample B. Finally, sample D contains an InGaN layer of $120 \mathrm{~nm}$ thickness and $21 \%$ Indium not grown on a MOVPE-GaN template.

\section{Results}

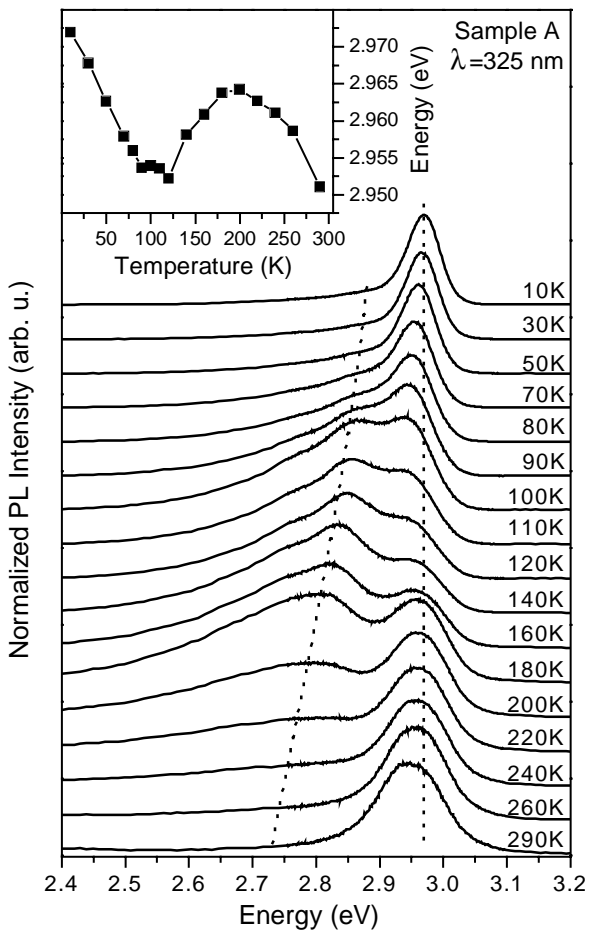

Fig. 1: PL spectra of sample $A$ at different temperatures. The inset shows the position of the high-energy peak vs. temperature.
Fig. 1 shows the temperature dependence of the photoluminescence of sample A. One can distinguish between two different PL peaks, which change their relative intensities with temperature. At very low temperatures $(10 \mathrm{~K}-70 \mathrm{~K})$ and at room temperature the high-energy peak dominates the spectra. Furthermore this luminescence exhibits a "S-shaped" emission position shift with temperature as can be seen in the inset of Fig. 1. This behavior (redshift-blueshift-redshift) was first explained by Cho et al. [11] in terms of inhomogeneity and carrier localization in the InGaN. Recently, it has been shown that the "S-shape" behavior becomes less pronounced with increasing excitation power which can also be understood in terms of local potential fluctuations in the InGaN [12]. We think that thermalization and carrier freeze out in potential fluctuations leads to this unexpected temperature behavior. In this meaning the high-energy peaks is not only one emission line, but the spatially integrated luminescence of locally different recombination energies, which mirrors the distribution of the potential fluctuations.

We assign the low-energy peak being dominant between $90 \mathrm{~K}$ and $180 \mathrm{~K}$ as recombination from electronic states deeper in the band gap. 

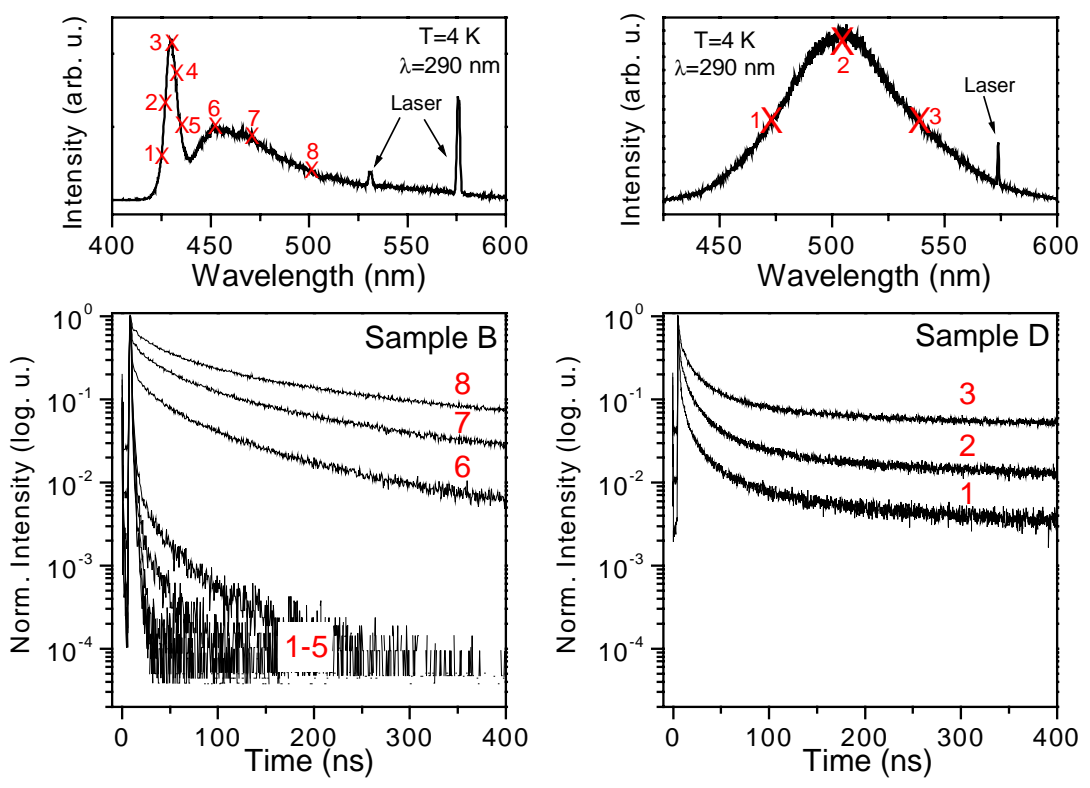

Fig. 2: Transients of sample B and D at different spectral positions (lower part) as indicated in the PL spectra (upper part).

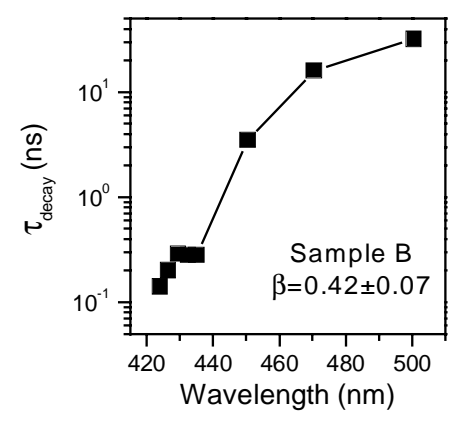

Fig. 3: Decay time as function of the emission wavelength for sample $B$.
To further investigate the underlying recombination processes we performed timeresolved PL experiments. Fig. 2 shows the result for the samples B (left hand side) and D (right hand side). In the upper part the PL spectra at $4 \mathrm{~K}$ are depicted and in the lower part the transients taken at the indicated spectral positions. In principle the PL spectrum of sample B is similar to those of sample $\mathrm{A}$ and $\mathrm{C}$, all grown on the MOVPE-GaN template. In contrast, the sample D exhibits only one broad luminescence structure. The temporal behavior of the luminescence is a multiple exponential decay for any spectral position and for all the samples. Pophristic et al. [13] claimed that this decay behavior can be described by $\exp \left[-(\mathrm{t} / \tau)^{\beta}\right]$, the so-called stretched exponential. With this function it is possible to describe the recombination dynamics in heavily disordered systems. In Fig. 3 the decay times as determined by this model are depicted for different spectral positions of sample B. The general trend -also for sample A and C- is an increase in decay time with the detection wavelength. We find decay times of 150-300 ps and 2-32 ns for the high-energy 


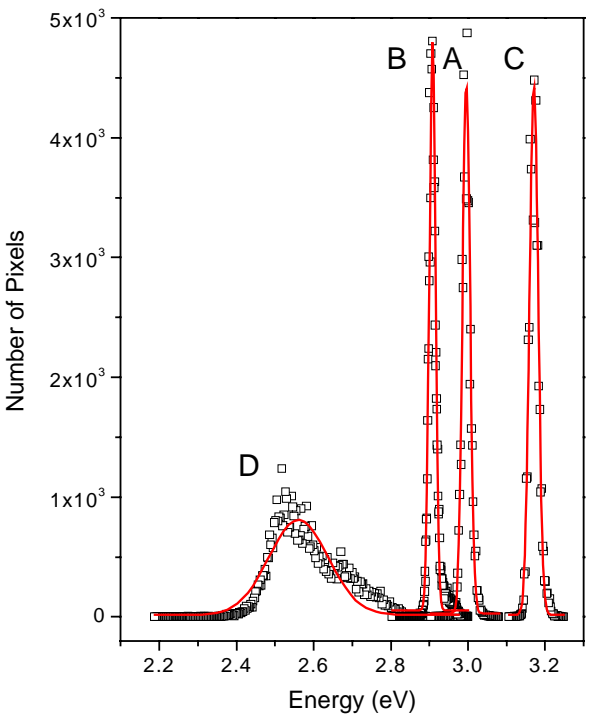

Fig. 4: CL histogramms of all four samples and the related Gaussian fit.

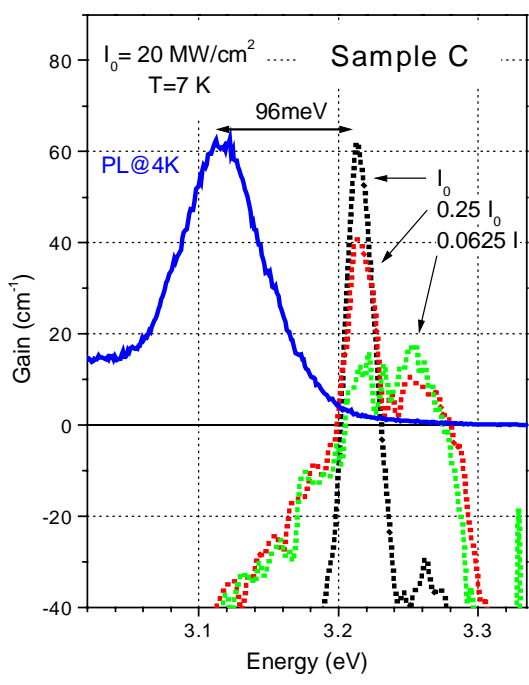

Fig. 5: Gain spectra of sample $C$ at $7 \mathrm{~K}$ for different excitation densities. For comparison a low-excitation PL is shown. and low-energy PL peak, respectively. The luminescence of sample D exhibits only long decay times in the ns range. It was shown that the decay times strongly depend on the excitation energy and intensity [14]. However, our results for the luminescence decay indicate that the samples under investigation are disordered. The disorder arises from local fluctuations in the Indium concentration leading to potential fluctuations. This is in line with the considerations about the thermodynamic growth conditions in the introductory part.

To achieve more information about the potential fluctuations we use the results of the CL measurements. Fig. 4 shows the histogramms of the four samples, i.e. the number of occurrence of an emission energy in the CL wavelength image of an area of $7 \mu \mathrm{m} \times 10 \mu \mathrm{m}$ ( $256 \times 200$ pixels) is depicted. For samples A-C we find relatively narrow Gaussian distributions, whereas for the sample D the plotted histogramm is much broader and nonGaussian. A Gaussian distribution indicates that the emission energies are random distributed on a lateral scale much smaller than the spatial resolution of the CL measurements $(45 \mathrm{~nm})$. For sample D the fluctuations are on a much larger scale, i.e. in the order of the spatial resolution or above. The difference between sample D and the others can not be explained by the higher Indium content of sample $\mathrm{B}$, since alloy broadening following $[\mathrm{x}(1-\mathrm{x})]^{1 / 2}$ can not explain the much broader distribution with a change in line shape. We think that the improvement from sample $\mathrm{D}$ to sample A-C is a result of better adjusted growth conditions and the use of the MOVPE-GaN templates.

In the following we will discuss how the degree of fluctuations affects the optical amplification. Fig. 5 shows the optical gain of sample $\mathrm{C}$ as function of the energy for different excitation energy. For 
comparison the low-intensity excitation PL is plotted. A gain structure between $3.2 \mathrm{eV}$ and $3.3 \mathrm{eV}$ appears for the lowest excitation. For higher power densities a sharp structure centered around $3.22 \mathrm{eV}$ strongly increases having a maximum gain value of $62 \mathrm{~cm}^{-1}$. This is assigned to the onset of lasing in the structure. The lasing peak is $96 \mathrm{meV}$ higher in energy than the PL maximum and can be explained by the filling of states due to very high excitation densities during the gain measurements. The correlation between optical gain and structural properties is given in Table 1.

\begin{tabular}{|c|c|c|c|c|}
\hline Sample & \multicolumn{2}{|c|}{ Gaussian Fit of CL Histogramms } & \multicolumn{2}{c|}{ Gain $(7 \mathrm{~K})$} \\
\hline & $\mathrm{E}_{\text {center }}(\mathrm{eV})$ & $\sigma$ & $\mathrm{E}_{\text {gain }}(\mathrm{eV})$ & $\mathrm{g}_{\max }\left(\mathrm{cm}^{-1}\right)$ \\
\hline $\mathrm{A}$ & 2.996 & 0.009745 & 3.131 & 37 \\
\hline $\mathrm{B}$ & 2.908 & 0.007895 & 2.899 & 10 \\
\hline $\mathrm{C}$ & 3.171 & 0.011265 & 3.213 & 62 \\
\hline $\mathrm{D}$ & 2.561 & 0.0805 & - & - \\
\hline
\end{tabular}

Table 1: Giving the results of the Gaussian fit of the CL histogramms (51200 data points) and the gain values at $7 \mathrm{~K}$. The $\sigma$ is the standard deviation.

We find that the samples A-C which exhibit a Gaussian CL histogramm show material gain at $7 \mathrm{~K}$. In contrast, sample D (grown without MOVPE-GaN template) has a broad non-Gaussian luminescence distribution and shows no optical amplification. The highest gain value is found for sample C, i.e. the $10 \times 5 \mathrm{~nm}$ MQW. We suggest that this is a result of confinement effects. On the other hand, the MQW has the broadest CL distribution of the three high-quality samples, which originates from Indium fluctuations but also from interface roughness [15]. However, on the small data basis of three samples we can not make a general statement on the dependence of $g_{\max }$ on $\sigma$ for the high-quality MBE samples.

\section{Conclusions}

We have investigated the optical and structural properties of InGaN/GaN heterostructures grown by MBE using time-resolved PL, CL microscopy and gain spectroscopy. For all the samples we found evidence for potential fluctuations. Particularly, the recombination dynamics resulting in transients, which can be fitted by stretched exponential decays, and the CL measurements are explained with this model. We found a Gaussian CL distribution and optical amplification for the samples grown on a MOVPE-GaN template. The best sample, a 10x5 nm MQW, shows gain values up to $62 \mathrm{~cm}^{-1}$ at $7 \mathrm{~K}$ and $10 \mathrm{~cm}^{-1}$ at $150 \mathrm{~K}$. Further improvement in growth conditions is expected to lead to optical amplification at RT, which is the requirement for laser fabrication.

\section{Acknowledgments}

The authors acknowledge the supply of the MOVPE-GaN templates on sapphire by R. Handschuh and B. Hahn (Osram Opto Semiconductors). A. Kaschner is gratefully for an Ernst-von-Siemens-scholarship. Work at Siemens/Infineon was partly supported by the German Ministry of Education and Research (BMBF). 


\section{References}

[1] S. Nakamura and G. Fasol: The Blue Laser Diode (Springer, Berlin, 1997).

[2] Y.-H Kwon, G. H. Gainer, S. Bidnyk, Y.-H. Cho, J. J. Song, M. Hansen, and S. P. DenBaars, Appl. Phys. Lett. 75, 2545 (1999).

[3] T. Wang, D. Nakagawa, M. Lachab, T. Sugahara, and S. Sakai, Appl. Phys. Lett. 74, 3128 (1999).

[4] H. Kollmer, J. S. Im, S. Heppel, J. Off, F. Scholz, and A. Hangleiter, Appl. Phys. Lett. 74, 82 (1999).

[5] I.-H. Ho and G. B. Stringfellow, Appl. Phys. Lett. 69, 2701 (1996).

[6] K. P. O’Donnell, R. W. Martin, and P. G. Middleton, Phys. Rev. Lett. 82, 237 (1999).

[7] R. Averbeck, A. Graber, H. Tews, D. Bernklau, U. Barnhöfer, and H. Riechert, SPIE Vol. 3279, 28 (1998).

[8] F. Bertram, T. Riemann, J. Christen, A. Kaschner, A. Hoffmann, C. Thomsen, K. Hiramatsu, T. Shibata, and N. Sawaki; Appl. Phys. Lett. 74, 359 (1999).

[9] K. L. Shaklee, R. E. Nahory, and R. F. Leheny, J. Lum. 7, 284 (1973).

[10] M. Schuster, P. O. Gervais, B. Jobst, W. Hösler, R. Averbeck, H. Riechert, A Iberl, and R. Stömmer, J. Phys. D Appl. Phys. 32, 56 (1999).

[11] Y.-H. Cho, G. H. Gainer, A. J. Fischer, J. J. Song, S. Keller, U. K. Mishra, and S. P. DenBaars, Appl. Phys. Lett. 73, 1370 (1998).

[12] J. Christen, T. Riemann, P. Fischer, J. Holst, A. Hoffmann, and M. Heuken, MRS Fall Meeting Boston 1999.

[13] M. Pophristic, F. H. Long, C. Tran, I. T. Ferguson, and R. F. Karlicek, J. Appl. Phys. 86, 1114 (1999).

[14] M. Pophristic, F. H. Long, C. Tran, R. F. Karlicek, Z. C. Feng, and I. T. Ferguson, Appl. Phys. Lett. 73, 815 (1998).

[15] Y.-H. Cho, J. J. Song, S. Keller, U. K. Mishra, and S. P. DenBaars,. Appl. Phys. Lett. 73, 3181 (1998). 